\title{
Viral infections in wheezing disorders
}

\author{
Peter Le Souëf
}

Affiliation: Faculty of Health and Medical Sciences, Dept of Respiratory Medicine, Child and Adolescent Health Service, University of Western Australia, Perth, Australia.

Correspondence: Peter Le Souëf, Division of Paediatrics, University of Western Australia, c/o Princess Margaret Hospital for Children, GPO Box D184, Perth, WA 6840, Australia. E-mail: peter.lesouefQuwa.edu.au

@ERSpublications

Viral infections in wheezing disorders in children http://ow.ly/qNPj30j38kS

Cite this article as: Le Souëf P. Viral infections in wheezing disorders. Eur Respir Rev 2018; 27: 170133 [https://doi.org/10.1183/16000617.0133-2017].

ABSTRACT Over the past year, studies into virus-induced wheeze in children have shifted towards investigations that examine the mechanisms by which respiratory viruses cause wheeze and an increase in studies examining the effects of novel interventions to reduce wheezing exacerbations. Studies on rhinovirus species (RV)-C infection have found that this is associated with a decrease in expression of CDHR3, the cellular receptor specific for this virus, and a decrease in interferon- $\beta$ expression, both of which are likely to favour RV-C infection. Recent clinical trials in children have found a decrease in wheezing exacerbations with both anti-respiratory syncytial virus antibody and anti-immunoglobulin $\mathrm{E}$ antibody therapy, and a clinical trial of prednisolone in children with their first RV-induced wheeze showed that only those with an $\mathrm{RV}$ viral count $>7000$ copies $\cdot \mathrm{mL}^{-1}$ responded. Further studies on the effects of bacterial lysates on immune system function continue to support the potential of this approach to reduce virus-induced wheezing exacerbations in children. These studies and many previous investigations into immunomodulation using bacterial lysates have led to the funding and commencement of a large study in which long-term administration of a bacterial lysate in young children will be assessed for its ability to prevent asthma.

\section{Introduction}

One of the challenges of treating and eventually preventing viral-induced wheezing in children is to understand the mechanisms involved from the inception of a respiratory viral infection to the onset and final resolution of an episode of wheeze. There have been few therapeutic advances over the past 50 years and they have made no real difference to the number of respiratory viral infections experienced by each child or to therapies to treat wheezing exacerbations. However, an area where there has been significant advance is in therapies that reduce the likelihood of a viral infection causing clinically significant wheeze. Studying the mechanisms by which these approaches successfully intervene in this process holds the potential for designing new and improved preventative measures. Researchers in this area might be inspired by Lord Kelvin, the famous 19th Century scientist [1] who is credited with saying: "to measure is to know". In the past year, several new studies have contributed to providing a better understanding of the mechanisms of viral-induced wheeze in children. Not all of these were mechanistic studies, but by introducing a new intervention using a novel approach that produces a clinical outcome, a mechanism is inferred and this points to the potential for more definitive studies to understand the key relevant, underlying mechanisms.

\section{Respiratory virus mechanisms of infection}

Respiratory viruses that produce wheeze in young children are common in the community, but the reasons why some cause more severe wheeze than others is not clear. A study of rhinovirus (RV) type C-infected

Received: Dec 122017 | Accepted after revision: March 152018

Provenance: Commissioned article, peer reviewed.

Copyright CERS 2018. ERR articles are open access and distributed under the terms of the Creative Commons Attribution Non-Commercial Licence 4.0. 
ciliated airway epithelial cells (AEC) in an air-liquid interface culture has provided new insight into why this virus type produces more severe clinical infections than the two other RV types, RV-A and RV-B [2]. Several key findings were made: 1) RV-C15 specifically targeted airway epithelial cells; 2) CDHR3, the cell receptor for RV-C was preferentially expressed on ciliated AEC; 3) inoculation of the culture with RV-C15 decreased CDHR3 expression; and 4) CDHR3 expression was decreased in cells in the culture that tested positive for RV-C15. These data provide excellent evidence that is consistent with RV-C using a yet unidentified mechanism to gain an immunological advantage by deceiving the host into decreasing expression of its human cell receptor. This could also occur passively if RV-C has a cytopathic effect on ciliated cells that express CDHR3. However, this experimental model does not rule out the alternative possibility that the human host is shutting down CDHR3 expression in RV-C infection to limit the spread or intensity of infection.

Several studies have shown that asthmatics have reduced innate immunity [3-6], but why this is so remains unclear. RV-C appears to be capable of interfering with the innate immune response. In another study of RV-C-infected AEC in an air-liquid interface culture, RV-C infection was found to be associated with a decrease in interferon (IFN)- $\beta$ mRNA and fluid concentration levels [7]. The ability to attenuate IFN- $\beta$ activation was ascribed to the RV-C $3 C^{\text {pro }}$ (a picornavirus protease and endopeptidase enzyme that cleaves peptide bonds of non-terminal sequence), and the 40 -His site of $3 C^{\text {pro }}$ was integral for this activity. The authors concluded that these findings are a plausible mechanism by which RV-C reduced innate immune function to facilitate its infectivity. However, an alternative explanation is that the human host is reducing excessive innate immune activity to limit the inflammatory response to infection.

\section{Immunisation to reduce respiratory viral infections}

If a natural infection does not produce reliable immunity against a further infection, developing an immunisation against that infection is likely to be difficult or impossible [8]. A problem also exists with infections where there are many strains if immunity from the natural infection only works for that strain, and this is a problem for attempting to develop a vaccine against rhinovirus. A possible way of combatting this was reported in a study in which a 25 -valent RV vaccine was tested in mice and a 50 -valent rhinovirus vaccine was tested in macaques [9]. These vaccines induced adequate neutralising antibody levels against the great majority of the RV types included in the vaccine and valency was not a major factor for determining the potency or breadth of response. One of the limitations of this study was that RV-C was not included among the types tested. Overall, the study suggests that successful vaccines may be possible for viruses even when there are a large number of strains. Whether these findings can be translated into clinical practice is not clear at present, but if it is possible to successfully vaccinate against all 150+ RV strains, wheezing exacerbations in children would be substantially reduced, given that several studies have shown that $>80 \%$ of such episodes are due to RV infection [10].

\section{Insights from clinical trials}

A follow-up of a cohort of children who received prednisolone or placebo for their first RV-induced wheezing illness has shown that those with a high rhinovirus viral load $\left(>7000 \mathrm{copies} \cdot \mathrm{mL}^{-1}\right)$ at time of infection were those that responded to prednisolone therapy with a decreased risk of requiring asthma medication over the subsequent 4 years [11]. This suggests that viral load of RV infection in early life has the potential to be used as a marker for those who will respond to treatment with oral steroids for their first RV-induced wheezing illness. The observation that this treatment can have long-term effects on reducing further exacerbations is unexpected and further studies to explore this observation more fully would be worthwhile.

In a Japanese multicentre observational, case-control study in preterm infants aimed at preventing respiratory syncytial virus (RSV), the anti-RSV monoclonal antibody palivizumab was given to 444 preterm infants 33-35 weeks gestational age, 349 received three of more doses of palivizumab during the first year of life and 95 received no palivizumab [12]. Of this cohort, 202 who had received treatment and 66 controls were followed to 6 years of age. There was no reduction in the number of children developing atopic asthma by 6 years of age, but the rate of recurrent wheeze during this period was reduced over two-fold in the palivizumab-treated versus the untreated group $(\mathrm{p}<0.001)$. These results are of relevance, as previous studies have suggested that RSV is a risk factor for the later development of asthma and allergy [13] and that anti-RSV monoclonal antibody prevents recurrent wheeze in an atopy-dependent manner [14]. Given that RSV infection is most prevalent in the first year or two of life [15], the Japanese study suggests that preventing infection in these early years has effects further into childhood, but that this effect is independent of atopy. More detailed studies to examine immune system responses to anti-RSV monoclonal antibody therapy are now needed for further progress to be made in understanding the role of RSV in wheeze and asthma. 
Biologics can be seen as highly focused interventions that specifically perturb one aspect of immune system function. As such, they are capable of providing excellent insight into the function of that pathway. In the PROSE (Preventative Omalizumab or Step-up Therapy for Severe Fall Exacerbations) study, a randomised study of the effects of omalizumab on rhinovirus infections and asthma, 259 allergic asthmatic children aged 6-17 years were administered the anti-immunoglobulin (Ig) E antibody and 89 controls were continued on within-guideline therapy [16]. The main findings were that those receiving omalizumab had: 1) a reduced RV prevalence in nasal mucus samples collected weekly for 3 months; 2) a decrease in peak viral shedding; 3) a reduced number and duration of "colds"; and 4) a reduced number of viral illnesses. This is the first direct evidence that anti-IgE decreases rhinovirus prevalence. Studies are now needed to determine why this occurs, as IgE is related to T-helper (Th)2 immunity, but Th2 responses are not considered to be directly involved in either protection from or susceptibility to virus infections [17].

Also in the PROSE study, the effect of reducing IgE with omalizumab on asthma exacerbations was assessed and specimens from study children were used to assess ex vivo plasma dendritic cell ( $\mathrm{pDC}$ ) antiviral interferon (IFN)- $\alpha$ responses [18]. The main findings were that omalizumab enhanced pDC antiviral IFN- $\alpha$ responses and attenuated pDC high affinity IgE receptor (FcERI) $\alpha$ protein expression. The combination of these results suggests that these effects are related and that allergic sensitisation plays a role in the observed increased susceptibility to virus-induced exacerbations of asthma.

\section{Prevention of asthma}

We know that the natural environment strongly influences asthma prevalence as asthma is rare in children in developing countries [19] and traditional farming communities [20]. Strong evidence implicates exposure to bacteria as the prime suspect, as endotoxin levels correlate inversely with asthma [20]. Also, bacterial lysates are known to reduce wheezing exacerbations in children [21,22] and reverse aspects of the relative degree of immunodeficiency in asthmatics [21]. Finally, recent microbiome data show clear links between gut flora and immunological programming $[23,24]$. Together this evidence raises the possibility that exposure to bacteria is a key factor in the lower rates of asthma reported in developing countries and rural communities and that administration of bacterial lysates in early life has the potential to prevent asthma developing.

However, the mechanisms driving this protective influence are still not well understood. Recent studies in mice have provided further evidence that bacterial lysates act as immunomodulating agents that can modify inflammatory responses. In a study of mouse bone marrow-derived dendritic cells (BMDC) [25], the bacterial lysate OM-85 was found to increase IFN- $\beta$ production and, while it did not activate the inflammasome itself, it primed BMDCs significantly increasing pro-interleukin (IL)- $1 \alpha$ and proIL- $1 \beta$ levels, in agreement with data in human dendritic cells [26]. The authors speculated that their results indicate that exposure to bacteria (or bacterial lysates) "keeps innate immune cells in an 'alerted state' ideal to release large amounts of IL-1 upon sensing an inflammasome trigger and, conceivably, to reduce selected viral infections" [25].

In another study of bacterial lysates in mice, the mice pre-treated with ovalbumin showed evidence that OM-85 decreased Th2 responses (IL-4), decreased eosinophils in bronchoalveolar lavage fluid (BALF) and increased Th1 (IFN- $\alpha$ ) levels in BALF [27]. In each case, OM-85 produced an enhanced response to that of a corticosteroid alone. These findings demonstrate that bacterial lysates shift the immune response away from those related to allergy and asthma and reinforces immune responses that enhance defence against viral infection.

In recognition of the potential importance of immunomodulation in the prevention of asthma, the National Institutes of Health has funded a large, multicentre phase 2 study [28]. Children aged 618 months will be recruited and receive OM- 85 or placebo for 24 months with an extended observation period following that to determine time to first wheezing lower respiratory illness, as well as number of respiratory illnesses.

\section{The role of the microbiome}

Whether disruption of the airway microbiome by respiratory viral infections is causal in the later development of asthma or a bystander event in a process driven by virus infections remains unclear. Two new studies have evaluated the airway microbiome after infection with RV. The first of these tested the hypothesis that RV infection alters the upper airway microbiota, and that microbial changes correspond with infection severity [29]. Children in this study were enrolled in the larger "RhinoGen" study and had nasal mucus samples collected weekly for 5 weeks in September, the peak RV season. For samples obtained before and after an asymptomatic RV infection, the latter specimen had a greater abundance of Dolosigranulum and Corynbacterium, whereas for samples obtained before and after an RV-induced wheezing/asthma exacerbation, the latter had an increased abundance of Moraxella and a decrease in 
Corynbacterium. The authors suggested a higher level of Dolosigranulum and Corynbacterium may confer protection from RV infection and that symptomatic RV infection may result in increases in Moraxella in the airway. In the second study, 29 families with at least two children were followed daily from the start of symptoms in any family member; children with an RV infection had a 4.3-fold increase in pneumococcal acquisition from the community and a 14.8-fold increase in within-family transmission of pneumococcus [30]. Whether the increase in pneumococcal transmission is likely to result in a clinically significant infection is unclear.

\section{The role of the virome}

The technical issues involved in assessment of the airway virome are still being resolved [31] and as yet, very few studies of the respiratory virome in children have been reported. In a study from Thailand of 103 children who had presented with an influenza-like illness, viruses detected by deep sequencing in nasopharyngeal specimens included many viruses that were not detected by Luminex assay [32]. In a study from China, analysis of the nasopharyngeal virome with multiplex metagenomic analyses using next-generation sequencing platform, children with a severe acute respiratory infection had a wide variety of viral species present including members of the Paramyxoviridae, Coronaviridae, Parvoviridae, Orthomyxoviridae, Picornaviridae, Anelloviridae and Adenoviridae families, whereas the viral population found in the respiratory tracts of children without severe acute respiratory infection was less diverse and mainly dominated by the Anelloviridae family with only a small proportion of common respiratory viruses [32]. These findings need to be replicated in other studies, but the finding that the virome is more diverse in children with a symptomatic infection contrasts with results on the bacterial microbiome that show a reduced diversity in those with a respiratory illness [33].

Conflict of interest: None declared.

\section{References}

1 Thompson SP. The life of Lord Kelvin (Originally published 1910, reissued 1976). 2nd Edn. Providence, AMS Chelsea Publishing/American Mathematical Society, 1976.

2 Griggs TF, Bochkov YA, Basnet S, et al. Rhinovirus C targets ciliated airway epithelial cells. Respir Res 2017; 18: 84.

3 Durrani SR, Montville DJ, Pratt AS, et al. Innate immune responses to rhinovirus are reduced by the high-affinity IgE receptor in allergic asthmatic children. J Allergy Clin Immunol 2012; 130: 489-495.

4 Edwards MR, Regamey N, Vareille M, et al. Impaired innate interferon induction in severe therapy resistant atopic asthmatic children. Mucosal Immunol 2013; 6: 797-806.

5 Wark PA, Johnston SL, Bucchieri F, et al. Asthmatic bronchial epithelial cells have a deficient innate immune response to infection with rhinovirus. J Exp Med 2005; 201: 937-947.

6 Johnston SL. Innate immunity in the pathogenesis of virus-induced asthma exacerbations. Proc Am Thorac Soc 2007; 4: 267-270.

7 Pang LL, Yuan XH, Shao CS, et al. The suppression of innate immune response by human rhinovirus C. Biochem Biophys Res Commun 2017; 490: 22-28.

8 Oyston P, Robinson K. The current challenges for vaccine development. J Med Microbiol 2012; 61: 889-894.

9 Lee S, Nguyen MT, Currier MG, et al. A polyvalent inactivated rhinovirus vaccine is broadly immunogenic in rhesus macaques. Nature Commun 2016; 7: 12838.

10 Bizzintino J, Lee W-M, Laing IA, et al. Association between human rhinovirus C and severity of acute asthma in children. Eur Respir J 2011; 37: 1037-1042.

11 Koistinen A, Lukkarinen M, Turunen R, et al. Prednisolone for the first rhinovirus-induced wheezing and 4-year asthma risk: a randomized trial. Pediatr Allergy Immunol 2017; 28: 557-563.

12 Mochizuki H, Kusuda S, Okada K, et al. Palivizumab prophylaxis in preterm infants and subsequent recurrent wheezing. six-year follow-up study. Am J Respir Crit Care Med 2017; 196: 29-38.

13 Sigurs N, Aljassim F, Kjellman B, et al. Asthma and allergy patterns over 18 years after severe RSV bronchiolitis in the first year of life. Thorax 2010; 65: 1045-1052.

14 Simoes EA, Carbonell-Estrany $\mathrm{X}$, Rieger $\mathrm{CH}$, et al. The effect of respiratory syncytial virus on subsequent recurrent wheezing in atopic and nonatopic children. J Allergy Clin Immunol 2010; 126: 256-262.

15 Brini I, Guerrero A, Hannachi N, et al. Epidemiology and clinical profile of pathogens responsible for the hospitalization of children in Sousse area, Tunisia. PLoS One 2017; 12: e0188325.

16 Esquivel A, Busse WW, Calatroni A, et al. Effects of omalizumab on rhinovirus infections, illnesses and exacerbations of asthma. Am J Respir Crit Care Med 2017; 196: 985-992.

17 Message SD, Johnston SL. The immunology of virus infection in asthma. Eur Respir J 2001; 18: 1013-1025.

18 Gill MA, Liu AH, Calatroni A, et al. Enhanced plasmacytoid dendritic cell antiviral responses after omalizumab. J Allergy Clin Immunol 2017; 18: in press [https://doi.org/10.1016/j.jaci.2017.07.035].

19 Annamalay AA, Lanaspa M, Khoo SK, et al. Rhinovirus species and clinical features in children hospitalised with pneumonia from Mozambique. Trop Med Int Health 2016; 21: 1171-1180.

20 Braun-Fahrlander C, Riedler J, Herz U, et al. Environmental exposure to endotoxin and its relation to asthma in school-age children. N Engl J Med 2002; 347: 869-877.

$21 \mathrm{Lu} \mathrm{Y,} \mathrm{Li} \mathrm{Y,} \mathrm{Xu} \mathrm{L,} \mathrm{et} \mathrm{al.} \mathrm{Bacterial} \mathrm{lysate} \mathrm{increases} \mathrm{the} \mathrm{percentage} \mathrm{of} \mathrm{natural} \mathrm{killer} \mathrm{T} \mathrm{cells} \mathrm{in} \mathrm{peripheral} \mathrm{blood} \mathrm{and}$ alleviates asthma in children. Pharmacology 2015; 95: 139-144.

22 Razi CH, Harmanci K, Abaci A, et al. The immunostimulant OM-85 BV prevents wheezing attacks in preschool children. J Allergy Clin Immunol 2010; 126: 763-769.

23 Velasquez-Manoff M. Gut microbiome: the peacekeepers. Nature 2015; 518: S3-S11. 
24 Gollwitzer ES, Marsland BJ. Microbiota abnormalities in inflammatory airway diseases - potential for therapy. Pharmacol Ther 2014; 141: 32-39.

25 Dang AT, Pasquali C, Ludigs $\mathrm{K}$, et al. OM-85 is an immunomodulator of interferon- $\beta$ production and inflammasome activity. Sci Rep 2017; 7: 43844.

26 Parola C, Salogni L, Vaira X, et al. Selective activation of human dendritic cells by OM-85 through a NF-kB and MAPK dependent pathway. PLoS One 2013; 8: e82867.

27 Liu C, Huang R, Yao R, et al. The immunotherapeutic role of bacterial lysates in a mouse model of asthma. Lung 2017; 195: 563-569.

28 University of Arizona. Oral Bacterial Extract for the Prevention of Wheezing Lower Respiratory Tract Illness (ORBEX). https://clinicaltrials.gov/ct2/show/NCT02148796 Date last updated: Jan 202017.

29 Kloepfer KM, Sarsani VK, Poroyko V, et al. Community-acquired rhinovirus infection is associated with changes in the airway microbiome. J Allergy Clin Immunol 2017; 140: 312-315.

30 Karppinen S, Terasjarvi J, Auranen K, et al. Acquisition and transmission of Streptococcus pneumoniae is facilitated during rhinovirus infection in families with children. Am J Respir Crit Care Med 2017; 196: 1172-1180.

31 Mitchell AB, Oliver BG, Glanville AR. Translational aspects of the human respiratory virome. Am J Respir Crit Care Med 2016; 194: 1458-1464.

32 Zhou Y, Fernandez S, Yoon IK, et al. Metagenomics study of viral pathogens in undiagnosed respiratory specimens and identification of human enteroviruses at a Thailand hospital. Am J Trop Med Hyg 2016; 95: 663-669.

33 Faner R, Sibila O, Agusti A, et al. The microbiome in respiratory medicine: current challenges and future perspectives. Eur Respir J 2017; 49: 1602086. 